Instituto Internacional de Investigación y Desarrollo Tecnológico Educativo INDTEC, C.A.

DOI: https://doi.org/10.29394/Scientific.issn.2542-2987.2021.6.22.17.332-344

OAl-PMH: http://www.indteca.com/ojs/index.php/Revista Scientific/oai

Artículo Original / Original Article

\title{
Gobierno digital y gestión municipal en la municipalidad de Tarma - Junín del periodo 2019-2020
}

Autora: Sofía Gabriela Cosquillo Lavado
Universidad Nacional Mayor de San Marcos, UNMSM

cosquillosofia@gmail.com

Lima, Perú

https://orcid.org/0000-0001-8643-494X

\section{Resumen}

Este este artículo, lo que se quiere es determinar como la Municipalidad Provincial de Tarma, se adapta a los avances tecnológicos que en la actualidad han aparecido, teniendo en cuenta el contexto en que nos encontramos por la pandemia del COVID-19, evitándose que la atención de la entidad sea de forma presencial, utilizando las herramientas tecnológicas para una mejor atención. Para lo cual, se desarrolló una plataforma digital que se llama Municipalidad de Tarma a tus Servicios, el cual permite acercar a la municipalidad con la ciudadanía. Esta propuesta permite a la entidad realizar una transformación de gobierno digital, realizando una gestión cero papel, con transparencia en sus acciones, brindando servicios administrativos de manera eficiente y eficaz, acelerando los trámites administrativos a favor de todos los ciudadanos, el método es la recolección de datos, es la entrevista y encuestas a los gobernantes de la Municipalidad Provincial de Tarma en Perú y a los pobladores.

Palabras clave: gobierno digital; transformación; ciudadanos; servicios digitales.

Código de clasificación internacional: 3311.01 - Tecnología de la automatización.

\footnotetext{
Cómo citar este artículo:

Cosquillo, S. (2021). Gobierno digital y gestión municipal en la municipalidad de Tarma - Junín del periodo 2019-2020. Revista Scientific, 6(22), 332-344, e-ISSN: 2542-2987. Recuperado de: https://doi.org/10.29394/Scientific.issn.2542-2987.2021.6.22.17.332-344
}

Fecha de Recepción: 29-07-2021
Fecha de Aceptación:

07-10-2021
Fecha de Publicación: 05-11-2021 
OAl-PMH: http://www.indteca.com/ojs/index.php/Revista Scientific/oai

Artículo Original / Original Article

\title{
Digital government and municipal management in the municipality of Tarma - Junín for the period 2019-2020
}

\begin{abstract}
In this article, what is wanted is to determine how the Provincial Municipality of Tarma, adapts to the technological advances that have appeared today, taking into account the context in which we find ourselves due to the COVID-19 pandemic, preventing the Attention to the entity is in person, using technological tools for better service. For which, a digital platform was developed called Municipality of Tarma to your Services, which allows the municipality to be brought closer to citizens. This proposal allows the entity to carry out a transformation of digital government, carrying out zero paper management, with transparency in its actions, providing administrative services efficiently and effectively, speeding up administrative procedures in favor of all citizens, the method is the collection of data, is the interview and surveys of the governors of the Provincial Municipality of Tarma in Peru and the inhabitants.
\end{abstract}

Keywords: digital government; transformation; citizens; digital services. International classification code: 3311.01 - Automation technology.

\footnotetext{
How to cite this article:

Cosquillo, S. (2021). Digital government and municipal management in the municipality of Tarma - Junín for the period 2019-2020. Revista Scientific, 6(22), 332-344, e-ISSN: 2542-2987. Recovered from: https://doi.org/10.29394/Scientific.issn.2542-2987.2021.6.22.17.332-344
}

Date Received: 29-07-2021
Date Acceptance:

07-10-2021
Date Publication: 05-11-2021 


\section{Introducción}

El trabajo permite que los ciudadanos pueda realizar el $100 \%$ de sus trámites sean de forma digital sin necesidad de acercarse a la Municipalidad Provincial de Tarma, Junín, en Perú, ya que la municipalidad son parte de la iniciativa Cero Papel del Ministerio del Ambiente de Perú, el cual su objetivo es contribuir con el desarrollo de una gestión pública, eficiente y eficaz, acelerando los trámites administrativos en los servicios que prestan a los ciudadanos, así mismo ya no habrá demoras en los servicios que brinda la municipalidad, en esa línea se reduce el malestar del ciudadano al realizar colas, tener los documentos físicos para la entrega, reduce tiempo, porque permite realizar cualquier trámite desde el lugar en el que se encuentre y lo más importante reduce actos de corrupción que se pueden generar al solicitar algún trámite o servicio dentro de la municipalidad.

La propuesta es realizar una plataforma digital el cual se llamará: Municipalidad de Tarma a Tus Servicios, el cual tiene como objetivo acercar la Municipalidad a la Ciudadanía; contiene cuatro componentes, las cuales son las siguientes:

- Orientación de trámites y servicios.

- Páginas institucionales.

- Servicios Digitales

- Reclamos.

Los tipos de servicios que se ofrece para la transformación digital en la Municipalidad Provincial Tarma, Junín, es la siguiente:

- Sin contacto: El ciudadano no tiene la necesidad de acercarse a la entidad para realizar los trámites administrativos, ya que el trámite lo podrá realizar desde el aplicativo, el cual tendrá acceso desde cualquier dispositivo electrónico inteligente. Este servicio es de manera automática y rápida. 
Instituto Internacional de Investigación y Desarrollo Tecnológico Educativo INDTEC, C.A.

DOI: https://doi.org/10.29394/Scientific.issn.2542-2987.2021.6.22.17.332-344

OAl-PMH: http://www.indteca.com/ojs/index.php/Revista Scientific/oai

Artículo Original / Original Article

- Semi presencial: El ciudadano realiza el trámite administrativo a través del aplicativo desde un dispositivo electrónico inteligente, sin embargo, por seguridad en la identificación del administrado, se requiere que se apersone para registrar su firma y/o huella digital, la misma que permitirá a la entidad mantener la seguridad de información respecto a los trámites que se requiera.

Para lograr la transformación digital en la Municipalidad Provincial de Tarma es importante contar con un marco normativo. La transformación digital nos coloca ante un nuevo contexto, el cual se va a necesitar implementar nuevas instrucciones el cual garantice, la seguridad de los datos, la correcta identificación digital de los ciudadanos, la autenticidad de los comprobantes de pago y/o firmas digitales, asimismo, mantener la constante actualización en los sistemas de transacciones digitales, generando una mayor seguridad y confianza en los ciudadanos que usan el aplicativo.

Por lo que, podemos observar que ya existe un marco normativo que permite el uso de las tecnologías digitales en la administración pública, así como el uso de datos que se recauda es la expresión de hechos, informaciones o conceptos, que se expresa de cualquier manera adecuada para su tratamiento, almacenamiento, comunicación e interpretación. Los datos son un componente estratégico de la utilización del Gobierno Digital en el Estado y de la gestión municipal.

Estamos ante el cambio de toda la forma de gobierno frente al nuevo concepto de cómo se gestiona los servicios del Estado con el uso de las tecnologías de información las cuales se pueden planificar, administrar con esta nueva herramienta que es la nueva base, que apoya y se aplican dentro de la utilización del uso de la administración pública la cual contribuye al uso intensivo de las Tecnologías de la Información y la Comunicación (TIC).

Según Rosales (2015): se debe realizar la audiencia pública para 
realizar el presupuesto participativo sobre la definición de los proyectos con la rendición de cuentas a los alcaldes y la asamblea general que otorga el plan estratégico participativo con la metodología de un método de moderación grupal (METAPLAN) con uso de tarjetas de colores como didáctica en los talleres.

Además para la Organización para la Cooperación y el Desarrollo Económicos (OCDE, 2019): hay un avance en participación proactiva de los usuarios en reformas de Gobierno Digital en la mayoría de los países, apertura por defecto de las dimensiones con mayor puntuación que refleja el impulso político de iniciativa de datos abiertos en el marco de reformas de Gobierno Digital, con iniciativa de datos que deben ser coordinadas a políticas que promuevan un sector público con datos, políticas integrales de datos como es el liderazgo que están en cierta medida ausentes en las iniciativas del Gobierno Digital, adopción limitada de mecanismos formales, directrices, herramientas y plataformas compartidas que implican la ausencia de medios o acciones en el logro de objetivos trazados en estrategias de Gobierno Digital.

\section{Metodología (Materiales y métodos)}

El presente estudio tipo aplicada con nivel explicativa, se enfoca en responder preguntas que importan a las comunidades y organizaciones. A través de un proceso colaborativo, un investigador aplicado: Recopila información de los ciudadanos y trabajadores de la Municipalidad provincial de Tarma.

Esta investigación no pretende crear conocimiento teórico; su propósito es proporcionar información práctica para la toma de decisiones basadas en evidencia de los resultados sobre si se ve beneficiada con los servicios del Gobierno Digital y gestión municipal.

El trabajo es importante puesto que tiene por objeto aproximar el Gobierno Digital y la municipalidad, es importante el uso del papel y su 
adecuado uso de la información en la administración pública; transformando y modernizando el estado, ello puede convertirse en la herramienta más eficiente y eficaz para prestar servicios rápidos y transparentes a los ciudadanos.

Delimitación temporal, el proyecto se realiza en el año 2019-2020, el cual se recogen los datos; delimitación espacial, se realiza dentro del distrito de Tarma de la provincia de Tarma en la Región Junín en Perú; y delimitación social, los ciudadanos están enmarcados en su participación, pero también el Estado peruano mediante sus estamentos que ofrecen servicios digitales en término de tramitaciones que agilizan y evitan la burocracia.

\section{Resultados (análisis e interpretación de los resultados)}

Los resultados de esta investigación son producto de la aplicación de la entrevista realizada a la población de la provincia de Tarma y a los trabajadores de la Municipalidad Provincial de Tarma en Perú.

Con relación al objetivo de la investigación en el que consiste en cómo la Municipalidad Provincial de Tarma pueda iniciar una transformación digital en sus trámites administrativos a fin tener mayor alcance al ciudadano tarmeño, y así este pueda realizar sus trámites a distancia, sin demoras, y con mayor acceso a la información. Como se puede apreciar en las entrevistas realizadas a los ciudadanos de Tarma en la tabla 1.

Tabla 1. Entrevistas a los ciudadanos de Tarma.

\section{Ciudadano $\mathrm{N}^{\circ} 1$}

¿Señalaría que los trámites administrativos son más prácticos con el Gobierno Digital?

Los trámites que más realizo son los permisos de funcionamiento para mi negocio, creo que con esta modalidad digital he podido realizarlo más rápido, ya que logre hacerlo desde mi casa.

Ciudadano $\mathbf{N}^{\circ} 2$

¿Afirmaría que la atención digital es Yo creería que sí, ya que agiliza los trámites eficiente? evitando colas, y podemos hacer seguimiento a 
Instituto Internacional de Investigación y Desarrollo Tecnológico Educativo INDTEC, C.A.

DOI: https://doi.org/10.29394/Scientific.issn.2542-2987.2021.6.22.17.332-344

OAl-PMH: http://www.indteca.com/ojs/index.php/Revista Scientific/oai

Artículo Original / Original Article

\begin{tabular}{|c|c|}
\hline & nuestras solicitudes \\
\hline \multicolumn{2}{|l|}{ Ciudadano $\mathbf{N}^{\circ} 3$} \\
\hline $\begin{array}{l}\text { ¿Confirma que el uso del servicio digital } \\
\text { agiliza el tiempo? }\end{array}$ & $\begin{array}{l}\text { Confirmo, ya que el sistema es quien se encarga } \\
\text { de tramitar las solicitudes, esto permite que sea } \\
\text { más rápido que las personas que lo hacían } \\
\text { usualmente }\end{array}$ \\
\hline \multicolumn{2}{|l|}{ Ciudadano $N^{\circ} 4$} \\
\hline $\begin{array}{l}\text { ¿Afirmaría que el servicio digital evita } \\
\text { casos de corrupción? }\end{array}$ & $\begin{array}{l}\text { Confirmo que si, un servicio digital nos permite } \\
\text { tener mayor acceso a la información y así poder } \\
\text { vigilar las acciones que cometen los } \\
\text { funcionarios. }\end{array}$ \\
\hline
\end{tabular}

Fuente: La Autora (2021).

El trabajador de la Municipalidad de Tarma busca en todo momento agilizar los trámites de los usuarios, con la ejecución de procedimientos digitales dirigidos a tal fin, por lo que se consideró interrogar a quienes son los involucrados de manera directa en gestionar las solicitudes de los ciudadanos, sobre aspectos importantes que se consideran en el impacto del gobierno digital en los ciudadanos, como se puede apreciar en la tabla 2.

Tabla 2. Entrevistas a los trabajadores de la Municipalidad de Tarma.

Trabajador $\mathrm{N}^{\circ} 1$

Señalaría que la implementación del Gobierno Digital es más eficiente?

Yo creería que si, el ciudadano tiene la información a la mano, así puede ver los requisitos que se necesitan para cada trámite, evitando demoras en sus solicitudes.

Trabajador $\mathbf{N}^{\circ} 2$

¿Considera que la cantidad de trámites digitales ha aumentado por parte del ciudadano?

Vemos que, con este sistema digital, los vecinos se han animado a realizar sus trámites, ya que tienen respuestas más rápidas y sin demoras, motivando que puedan formalizarse. 


\begin{tabular}{|c|c|}
\hline \multicolumn{2}{|l|}{ Trabajador $\mathrm{N}^{\circ} 3$} \\
\hline $\begin{array}{l}\text { ¿Afirmaría que con el Gobierno Digital } \\
\text { existe mayor acceso a la información del } \\
\text { ciudadano común? }\end{array}$ & $\begin{array}{l}\text { Confirmo, las personas al tener la información } \\
\text { a la mano pueden acceder a todos los trámites } \\
\text { disponibles, asimismo en dicho sistema } \\
\text { también se publica los proyectos que realiza } \\
\text { la municipalidad, evidenciando en que se está } \\
\text { gastando los recursos a beneficio de todos los } \\
\text { tarmeños, esta información permite que las } \\
\text { personas puedan verificar el buen uso de sus } \\
\text { recursos }\end{array}$ \\
\hline \multicolumn{2}{|l|}{ Trabajador $N^{\circ} 4$} \\
\hline $\begin{array}{l}\text { ¿Afirmaría que los ciudadanos que tienen } \\
\text { acceso a la información pueden hacer } \\
\text { seguimiento a los trámites realizados? }\end{array}$ & $\begin{array}{l}\text { Claro que si, los ciudadanos tienen acceso a } \\
\text { toda la información de los trámites realizados, } \\
\text { el cual podrá hacer seguimiento y saber a } \\
\text { tiempo si es que existe alguna observación, } \\
\text { asimismo podrá ver todos los requisitos que } \\
\text { se necesitan para iniciar sus solicitudes }\end{array}$ \\
\hline
\end{tabular}

Fuente: La Autora (2021).

Los beneficios de los trámites y servicios digitales en todo lo que es el relacionamiento entre el gobierno y la ciudadanía y en lo que es el acceso a información pública y la transparencia de la disponibilidad de esa información.

Una dificultad de la Municipalidad de Tarma, antes de la digitalización, implicaba obligar a las personas, a trasladarse al lugar de atención eventualmente con traslados muy lejanos, algunos de los anexos, centros poblados, teniendo en cuenta a geografía donde están ubicados, y solo para hacer un trámite.

Otra dificultad que se asocia, es la falta de transparencia. Muchas veces no se sabía los requisitos se requieren para realizar su trámite, lo que generaba que el ciudadano tendría que hacer varios viajes para realizar su trámite, lo cual generaba un desgaste económico y físico, ya que muchas de estas personas se trasladan a pie. Asimismo, la calidad de servicio era irregular, demasiado dependiente de la persona de turno, se generaba largas colas, demoras, y costos asociados a esa calidad de servicio irregular. 
Entonces para solucionar los problemas, como se ha mencionado en los párrafos anteriores, se rediseñó los trámites y servicios más recurrentes, pensando siempre en el ciudadano de a pie, teniendo como principal objetivo dar una herramienta útil, que pueda facilitar el acceso a todos los tarmeños, vengan de donde vengan, pensando siempre desde su condición socioeconómica y no en los intereses particulares de quizás de algún funcionario.

Es así que, se debe utilizar las tecnologías para facilitar el desarrollo social y económico. Actualmente, gracias a la globalización más del $90 \%$ de las personas de la provincia de Tarma, tienen acceso a un dispositivo móvil, el cual tiene acceso a internet y distintas aplicaciones, es por ello, en busca de una solución para atender a aquellos ciudadanos tengan un mejor acceso a la información y a agilizar su trámites, es que desarrolla un sistema digital a través de los dispositivos móviles, dispositivo que en la mayoría de los ciudadanos tienen a la mano y puedan tener acceso a realizar lo que ellos necesiten.

No se trata de hacer online lo mismo que se estaba haciendo en papel, hay que rediseñar los procesos, simplificar los trámites. Los trámites más recurrentes deben estar más simplificados, que a través de unos minutos el ciudadano pueda contar con su certificado, constancia, etc., la misma que está registrada en una base de datos como parte del archivo digital de la Municipalidad Provincial de Tarma, Junín.

El aplicativo inicia con los trámites más concurrentes, sin embargo, el plan es asumir digitalizar la totalidad de los trámites, con esto conseguimos la disponibilidad de trámites en toda la Municipalidad, lo que más adelante se aplicará en todo el Estado, provocando un ahorro de costo por la eficiencia en la parte operativa del desarrollo masivo que se genera.

Así mismo el ciudadano tiene la confianza en sí mismo para hacer los trámites vía web, ya que su información va a ser manejada adecuadamente, 
el sistema está disponible las 24 horas, los 365 días del año.

Eso implica desde el punto de vista del Estado una necesidad de sensibilización con el ciudadano, aplicando estrategias de gestión mediante una planificación organizada como proceso cambio en la Gestión Municipal, el cual construirá un mundo moderno y tecnológico. En consecuencia, podemos indicar que el Gobierno Digital contribuye a la gestión de la Municipalidad Provincial de Tarma, Junín, a un desarrollo sostenible continuo.

Estamos en concordancia con el autor Pacheco (2017): quien indica que, para lograr una gestión digital, es necesario contar con el apoyo de las instituciones públicas y privadas, las cuales deben sumar sus fuerzas para facilitar el acceso, la asequibilidad y la utilización de las TIC, de esa manera se podrá realizar la modernización, en el cual se podrá contar con la información en tiempo real de gestión de la entidad.

De acuerdo con la Cooperación y el Desarrollo Económicos (OCDE, 2016): en su publicación sobre las políticas digitales estatales dentro de un panorama de mundialización y las maneras que se deben adoptar, afirma que: Un gobierno abierto es una forma de interacción sobre los servicios que otorga un gobierno local con transparencia dentro de un contexto diferente en cada país que es basado en políticas públicas y prácticas innovadores que son sostenibles en base a la rendición de cuentas con participación.

Esta información va de la mano con el principio de transparencia el cual toda entidad debe aplicar, es por ello que creemos que el Gobierno Digital aporta mucho en recuperar la confianza entre el ciudadano y la gestión municipal, teniendo en cuenta que dicha confianza ha sido vulnerada en muchas oportunidades por las gestiones anteriores, el cual no brinda la información a su población, su procedimiento administrativos son lentos e ineficientes, sin contar con los actos de corrupción que pudieron haber ocurrido.

Es por eso la importancia de esta herramienta digital, la cual permite 
realizar un control concurrente con su administración, generando así una mayor participación de los ciudadanos con su provincia. Además, reforzamos lo afirmado por los autores Estévez y Janowsk (2016): quienes indica que para ejecutar el Gobierno Digital se debe contar con una iniciativa de las empresas públicas y privadas.

Finalmente estamos completamente de acuerdo con Toro-García, Gutiérrez-Vargas y Correa-Ortiz (2020): quienes afirman que el uso de la administración electrónica en diferentes estados ayuda acercar a los ciudadanos al Estado, al incrementando la confianza en la modernización de los servicios que se brindan a los ciudadanos, es eso lo que podemos indicar que en la ciudad de Tarma podrá convertirse en una ciudad inteligente ya que la transformación digital será muy beneficioso para toda la población, ya que toda la información estará disponible en la plataforma digital.

\section{Conclusiones}

Concluimos que el Gobierno Digital en la Provincia de Tarma permitirá brindar los servicios administrativos de manera eficiente y eficaz, para los ciudadanos. Así mismo, la transparencia que brinda el Gobierno Digital, permitirá a los ciudadanos contar con la información de los recursos que administra la Municipalidad, así como también los trámites administrativos que ellos realizan a través de los servicios digitales.

No obstante, el Gobierno Digital se deberá solicitar la colaboración del sector público o privado, ya que es importante unir fuerzas para lograr la transformación de Tarma a un Gobierno Digital.

Finalmente, concluimos que las tecnologías digitales, permitirá al ciudadano contar con la tecnología necesaria para lograr acelerar los trámites administrativos, tener acceso a los servicios que ofrece la municipalidad reduciendo brechas entre el ciudadano y el estado. 


\section{Referencias}

Estévez, E., \& Janowsk, T. (2016). Gobierno Digital, Ciudadanos y Ciudades Inteligentes. Revista Institucional de la Facultad de Informática, (3), 11-13, e-ISSN: 2468-9564. Recuperado de: http://sedici.un|p.edu.ar/handle/10915/53440

OCDE (2016). Gobierno Abierto: Contexto mundial y el camino a seguir. Francia: Cooperación y el Desarrollo Económicos.

OCDE (2019). Índice de Gobierno Digital OCDE 2019: Resultados y mensajes clave. Francia: Cooperación y el Desarrollo Económicos.

Pacheco, G. (2017). Retos y desafíos de la inclusión digital en el Perú. Congreso Internacional de Ingeniería Informática. Perú: Pontificia Universidad Católica del Perú. Recuperado de:

https://repositorio.pucp.edu.pe/index/handle/123456789/71262

Rosales, M. (2015). Una Guía para el Ejecutivo Eficaz: Los secretos del buen alcalde. (Edición actualizada). Chile: IULA/SACDEL, Servicios de Asistencia y Capacitación para el Desarrollo Local, de la Unión Internacional de Municipalidades.

Toro-García, A., Gutiérrez-Vargas, C., \& Correa-Ortiz, I. (2020). Estrategia de gobierno digital para la construcción de Estados más transparentes y proactivos. Trilogía Ciencia Tecnología Sociedad, 12(22), 71-102, e-ISSN: 2145-7778. Recuperado de:

https://doi.org/10.22430/21457778.1235 


\section{Sofía Gabriela Cosquillo Lavado}

e-mail: cosquillosofia@gmail.com

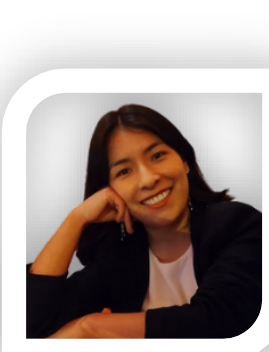

Nacida en Tarma del departamento de Junín, Perú, el 23 de febrero del año 1994. Ingeniera de Sistemas y Gestión de Tecnologías de Información por la Universidad Femenina del Sagrado Corazón (UNIFE); y Egresada de la Maestría en Gestión Pública por la Universidad Nacional Mayor de San Marcos (UNMSM), sede Lima, Perú, desde el año 2014; Especialista en Contrataciones del Estado; entre las principales actividades desarrolladas esta realizar procesos de contratación enfocados en la transparencia pública en contrataciones con el estado, de esa manera reducir la corrupción, realizando procesos con eficiencia y eficacia.

El contenido de este manuscrito se difunde bajo una Licencia de Creative Commons ReconocimientoNoComercial-Compartirlgual 4.0 Internacional 